Article

\title{
Freedericksz Transitions in Twisted Ferronematics Subjected to Magnetic and Laser Field
}

\author{
Cristina Cirtoaje ${ }^{1,+}+\mathbb{C}$, Gabriela Iacobescu ${ }^{2, *,+}(\mathbb{C}$ and Emil Petrescu $1,+(\mathbb{C}$ \\ 1 Department of Physics, The Faculty of Applied Sciences, University Politehnica of Bucharest, \\ 060042 Bucharest, Romania; cristina.cirtoaje@upb.ro (C.C.); emil.petrescu@upb.ro (E.P.) \\ 2 Department of Physics, Faculty of Sciences, University of Craiova, 200585 Craiova, Romania \\ * Correspondence: gabriela.iacobescu@edu.ucv.ro \\ + These authors contributed equally to this work.
}

Received: 19 May 2020; Accepted: 29 June 2020; Published: 1 July 2020

\begin{abstract}
The influence of the anchoring forces on the Freedericksz transition in twisted ferronematics simultaneously subjected to magnetic and laser fields is studied in this work. Using the elastic continuum theory and Gouchen model for molecular anchoring on the cell support plates, the critical field and the saturation field were calculated as a function of the laser intensity and anchoring strength for two types of ferronematics based on 5CB and CCN-37 liquid crystals.
\end{abstract}

Keywords: twisted ferronematics; Freedericksz transition; magnetic and laser fields

\section{Introduction}

Initially developed for the display industry as LCDs, where they seem to have reached their maximum potential, liquid crystals (LC) have now been reinvented due to the discovery of nanomaterials leading to new systems such as QLED (Quantum dot display) or OLED (Organic led). These technologies are consequences of years of research into nanoparticle properties. In LC research fields, many studies have been performed on magnetic nanoparticle dispersions in nematic phases, called ferronematics [1-12]. There is also interest in other particles and their effect on the molecular order of the host [13-31]. Usually, research works have focused on the phenomena which occur inside the system, and less work has focused on its edges. This has led to a loss of information regarding the parameters that are affected by the boundary conditions. In this manuscript, we present the influence of boundary anchoring forces on the critical and saturation fields of magnetic Freedericksz transitions, also considering the effect of the laser radiation used for observation.

When a liquid crystal is subjected to external stimuli (such as electric magnetic or laser fields), a molecular reorientation occurs. This is the Freedericksz transition, and the minimum value of the field intensity when this phenomenon appears is called the critical field. The field value at which all the molecules are reoriented according to the field direction and their magnetic or electric anisotropy is called the saturation field. These field values were calculated for two nematic liquid crystals used as hosts for magnetic nanoparticles: 5CB (4-Cyano-4'-pentylbiphenyl), which has a positive magnetic anisotropy, and CCN-37 ( $4 \alpha, 4^{\prime} \alpha$-propylheptyl- $1 \alpha, 1^{\prime} \alpha$-bicyclohexyl- $4 \beta$-carbonitrile), for which magnetic anisotropy is negative.

For a ferronematic composite, we consider rod-like magnetic nanoparticles. Burylov\&Co $[9,13]$ presented a detailed method to describe the liquid crystal molecule anchoring on ferromagnetic particles and calculated the interaction energy between these particles and the nematic host. A similar method can be used for ferroelectric particles or for any other long microparticle suspensions in LC. In our previous experimental research performed on ferromagnetic particles such as $\mathrm{CoFe}_{3} \mathrm{O}_{4}$ [30], we found that magnetic nanoparticles gather together, forming chains (about $2 \mu \mathrm{m}$ length) when mixed 
in nematics; thus, the use of cylinder magnetic particles for theoretical characterization is feasible. The proposed model in this paper will therefore consider a nematic liquid crystal, with cylindrical ferroparticles inserted in it, confined between two solid walls. When applying the elastic continuum theory [32], we should take into account all the interactions in this system: the elastic interaction between molecules, the interaction between nanoparticles and LC molecules, the interaction with the external field and (usually neglected in other papers) the interaction with the surface.

According to Rapini and Papoular's model [33], the surface anchoring energy of LC molecules on the boundary solid walls is

$$
F_{S}=\frac{1}{2} S A \sin ^{2} \theta_{0}
$$

where $A$ is the anchoring strength, $\theta_{0}$ is is the angle between the direction of easy axe and nematic director and $S$ is area of the contact surface between the nematic sample and the solid support.

Several years later, Guochen and collaborators [34] found a more complex formula for the surface energy:

$$
F_{S}=\frac{1}{2} S A \sin ^{2} \theta_{0}\left(1+\xi \sin ^{2} \theta_{0}\right)
$$

where $\xi$ is a parameter found by the authors to be -0.20 . Considering this formula and Burylov's theory for the interaction between the ferromagnetic nanoparticles and nematic host, critical and saturation fields were calculated and their dependencies of anchoring strength were plotted. For the experimental evaluation of the Freedericksz transition, a laser beam is used to detect the molecular reorientation. Depending on the crystal anisotropy and on the external field orientation, this laser beam may either increase or decrease the critical values by inducing a secondary Freedericksz transition. As can be seen from this manuscript, the laser beam's influence on these values can be significant if a high-intensity ray is used.

\section{Theoretical Considerations}

Using the surface anchoring energy given in [34], we can determine the free energy density of a ferronematic subjected to a magnetic and a laser field confined between two solid walls:

$$
F=S \int_{-d}^{+d} f_{v} d z+2 F_{S}
$$

where

$$
f_{v}=f_{v 1}+f_{v 2}+f_{v 3}+f_{v 4}+f_{v 5}
$$

is the free energy density in the bulk of the system, while the second term represents the anchoring energy on the solid edges.

For a proper evaluation of each term, the system must be established. A liquid crystal composite with ferromagnetic particles is confined between two solid walls, previously prepared for planar alignment. To obtain the twisted configuration, the rubbing directions on the plates are perpendicular to each other, meaning that the molecules rotate around the $\mathrm{Oz}$ axes with an angle of $\varphi$ that increases from 0 (on the bottom plate) to $\pi / 2$ (on the top one). In the absence of any external field (magnetic or laser), the ferromagnetic nanoparticles gather in long chains parallel with the nematic molecules' long axes (Figure 1a), as shown in previous research [2,12]. When a magnetic field is applied to the $\mathrm{Oz}$ direction, it acts both on nematic and ferroparticles. If a positive anisotropy liquid crystal is used, the LC-molecules tend to follow the field direction and a distortion angle $\theta$ is created with the Oxy plane. The value of the distortion angle depends on the field intensity: it tends to zero if the field magnitude is equal to the critical threshold for the Freedericksz transition, and it reaches its maximum value when the field intensity is equal to the saturation field. After this value, no other variation of $\theta$ occurs. On the other side, the field action on the ferroparticle is intense and the ferromagnetic chains are aligned parallel to the applied field. However, the anchoring forces between the LC-molecules 
and inserted particles prevent this alignment, and another distortion angle $(\beta)$ appears between the ferromagnetic chain and $\mathrm{Oz}$ axis. A laser beam must be sent through the sample to show the refractive index variation induced by the molecular director reorientation. If the laser intensity is sufficiently high, it can also affect the molecular orientation and thus the Freedericksz transition. To simplify the theoretical model, we consider the laser effect included in the same distortion angles $(\theta$ and $\beta$ ); these variables are discussed in the following part of this paper. Finally, we may conclude that when all the external stimuli are applied, a stable configuration is obtained, as represented in Figure $1 \mathrm{~b}$.

In Equation (4), the term $f_{v 1}$ is the liquid crystal's free energy contribution from the intermolecular elastic interaction.

$$
f_{v 1}=\frac{1}{2} K_{1}(\nabla \vec{n})^{2}+\frac{1}{2} K_{2}(\vec{n} \nabla \times \vec{n})^{2}+\frac{1}{2} K_{3}[\vec{n} \times(\nabla \times \vec{n})]^{2}
$$

where $K_{1}, K_{2}, K_{3}$ are the splay, twist and bend elastic constant, respectively.

The second term,

$$
f_{v 2}=-\frac{1}{2} \mu_{0}^{-1} \chi_{a}(\vec{B} \vec{n})^{2},
$$

represents the free energy density of the action of the magnetic field $\vec{B}$ on liquid crystal molecules, where $\mu_{0}$ is permeability of free space and $\chi_{a}$ is the magnetic anisotropy.

The term denoted by $f_{v 3}$ is the free energy density of the magnetic field's action on nanoparticles [14].

$$
f_{v 3}=-f M_{s} \vec{m} \vec{B}
$$

where $f$ is the volumic fraction of the magnetic particles, $M_{s}$ is the saturation magnetization of the nanoparticle substance and $\vec{m}$ is the unit vector of the ferromagnetic nanoparticles.

By $f_{v 4}$, we denote the free energy density of the molecular interaction with inserted nanoparticles $[9,13]$.

$$
f_{v 4}=\frac{W f}{a}(\vec{m} \vec{n})^{2}
$$

where $W$ is the surface density of the anisotropic part of the interfacial energy of the nanoparticle nematic boundary and $a$ is the ferromagnetic nanoparticle's diameter.

Finally, $f_{v 5}$ is the free energy density due to the interaction with the electromagnetic field given by Pieranski in [35] and discussed in [36]. Unlike the cited reference, we used $\theta$ as the angle between the molecular director and the solid walls instead of the angle made by molecular director with the laser wave vector.

$$
f_{v 5}=-I \frac{n_{0} n_{e}}{\sqrt{n_{0}^{2} \cos ^{2} \theta+n_{e}^{2} \sin ^{2} \theta}}
$$

where $n_{0}$ is the ordinary refractive index and $n_{e}$ is the extraordinary refractive index. $I$ is the mean volume density of the electromagnetic energy of the laser beam. The connection with the laser beam intensity $J$ is $J=c I$, where $c$ is the light speed in free space.

The twisted ferronematic is considered to be confined between two identical solid walls, parallel to the Oxy plane, placed at $z=-d$ and $z=d$. The applied field, $\vec{B}$, is parallel to the $\mathrm{Oz}$ axis. The nematic director $\vec{n}$ and the magnetic moment unit vector $\vec{m}$ are characterized by the polar angles $\theta, \beta$ and the azimuthal angles (the twist angles) $\varphi$ and $\gamma$, respectively (Figure 1). The boundary conditions are $\varphi=0$ for $z=-d$ and $\varphi=\pi / 2$ for $z=d$. 

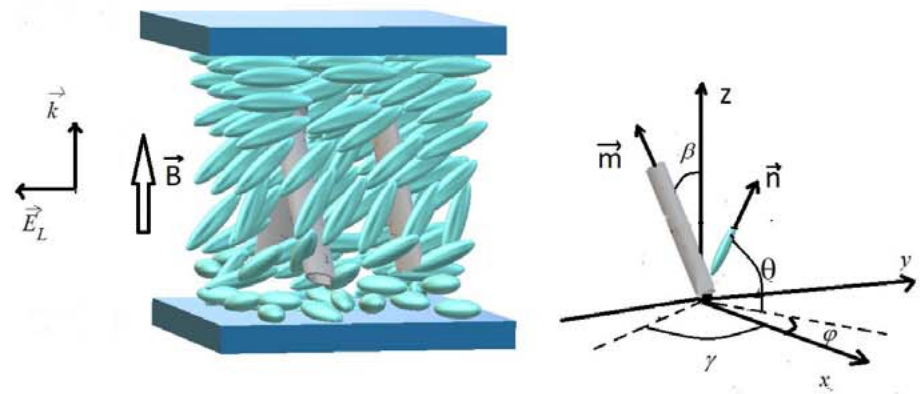

Figure 1. Molecular orientation in a twist subjected to a magnetic field $B$ and to a laser field with wave vector $k$ and electric component $E$.

In the presence of a distorting magnetic field, these angles are changed, with different values for different $z$ coordinates, and so the two vectors have the following components:

$$
\begin{array}{ll}
n_{x}=\cos \theta \cos \varphi & m_{x}=\sin \beta \cos \gamma \\
n_{y}=\cos \theta \sin \varphi & m_{y}=\sin \beta \sin \gamma \\
n_{z}=\sin \theta & m_{z}=\cos \beta
\end{array}
$$

where $\beta$ and $\theta$ angles appear as a result of the interaction of magnetic nanoparticles with liquid crystal molecules.

Considering the geometry given in Figure 1, Equations (5)-(8) become

$$
f_{v 1}=\frac{1}{2}\left(K_{1} \cos ^{2} \theta+K_{3} \sin ^{2} \theta\right) \dot{\theta}^{2}+\frac{1}{2} \cos ^{2} \theta\left(K_{2} \cos ^{2} \theta+K_{3} \sin ^{2} \theta\right) \dot{\varphi}^{2}
$$

where $\dot{\theta}=d \theta / d z$ and $\dot{\varphi}=d \varphi / d z$.

$$
\begin{gathered}
f_{v 2}=-\frac{\chi_{a}}{2 \mu_{0}} B^{2} \sin ^{2} \theta \\
f_{v 3}=-M_{s} f B \cos \beta \\
f_{v 4}=f \frac{W}{a}(-\cos \theta \cos \varphi \sin \beta \cos \gamma-\cos \theta \sin \varphi \sin \beta \sin \gamma+\sin \theta \cos \beta)^{2}
\end{gathered}
$$

From the Euler-Lagrange equation for $\gamma$, we obtain

$$
\frac{d}{d z}\left(\frac{\partial f_{v}}{\partial \dot{\gamma}}\right)-\frac{\partial f_{v}}{\partial \gamma}=0
$$

where $\dot{\gamma}=d \gamma / d z$; thus, we get $\gamma=\varphi$. The free energy density term depending on $\beta$ is therefore

$$
f_{\beta}=\frac{f W}{a} \sin ^{2}(\theta-\beta)-M_{s} f B \cos \beta
$$

The second Euler-Lagrange equation only considers the term depending on $\beta$, so the free energy density term depending on $\beta$ becomes

$$
\frac{d}{d z}\left(\frac{\partial f_{\beta}}{\partial \dot{\beta}}\right)-\frac{\partial f_{\beta}}{\partial \beta}=0
$$

where $\dot{\beta}=d \beta / d z$.

From Equation (17), we obtain

$$
\sin \beta=\frac{W}{a M_{s} B} \sin 2(\theta-\beta)
$$


Taking into account the magnitude orders for $M_{s}, W$ and $a\left(M_{s}=10^{4} \mathrm{~A} / \mathrm{m}, B>10^{-3} \mathrm{~T}\right.$, $W=5 \times 10^{-9} \mathrm{~N} / \mathrm{m}$ and $\left.a=10^{-8} \mathrm{~m}\right)[9,36], \beta$ is very small, and so

$$
f_{\beta}=\frac{f W}{a} \sin ^{2} \theta-M_{s} f B
$$

The Euler-Lagrange equations for $\varphi$ and $\theta$ have the following prime integrals:

$$
\begin{gathered}
\cos ^{2} \theta\left(K_{2} \cos ^{2} \theta+K_{3} \sin ^{2} \theta\right) \dot{\varphi}=C_{1} \\
\frac{1}{2}\left(K_{1} \cos ^{2} \theta+K_{3} \sin ^{2} \theta\right) \dot{\theta}^{2}-\frac{1}{2} \cos ^{2} \theta\left(K_{2} \cos ^{2} \theta+K_{3} \sin ^{2} \theta\right) \dot{\varphi}^{2} \\
+\frac{1}{2}\left(\frac{\chi_{a}}{\mu_{0}} B^{2}-\frac{2 f W}{a}\right) \sin ^{2} \theta+I \frac{n_{0} n_{e}}{\sqrt{n_{0}^{2} \cos ^{2} \theta+n_{e}^{2} \sin ^{2} \theta}}=C_{2}
\end{gathered}
$$

where $C_{1}$ and $C_{2}$ are constant for any $\theta$ and $\varphi$ and can be evaluated from boundary conditions.

\section{Critical Field}

When the magnetic field is just above the critical field, the deviation angle $\theta$ is very small; thus, by using a proper approximation, the prime integral of $\varphi$ given in Equation (20) becomes

$$
K_{2} \dot{\varphi}=C_{1}
$$

Considering the twisted nematic configuration, with $\varphi=0$, at $z=-d$, and $\varphi=\pi / 2$ at $z=d$, we get

$$
\varphi=\frac{\pi}{4}\left(\frac{z}{d}+1\right)
$$

Considering $\theta$ to be small, we then obtain

$$
I \frac{n_{0} n_{e}}{\sqrt{n_{0}^{2} \cos ^{2} \theta+n_{e}^{2} \sin ^{2} \theta}}=I n_{e}\left(1-\frac{N_{1}}{2} \sin ^{2} \theta+\frac{3}{8} N_{1}^{2} \sin ^{4} \theta\right)
$$

where

$$
N_{1}=\frac{n_{e}^{2}}{n_{0}^{2}}-1
$$

The other prime integral is

$$
\begin{aligned}
& \frac{1}{2}\left(K_{1} \cos ^{2} \theta+K_{3} \sin ^{2} \theta\right) \dot{\theta}^{2}-\frac{1}{2} \cos ^{2} \theta\left(K_{2} \cos ^{2} \theta+K_{3} \sin ^{2} \theta\right)\left(\frac{\pi}{4 d}\right)^{2} \\
& +\frac{1}{2}\left(\frac{\chi_{a}}{\mu_{0}} B^{2}-\frac{2 f W}{a}\right) \sin ^{2} \theta+I_{e}\left(1-\frac{N_{1}}{2} \sin ^{2} \theta+\frac{3}{8} N_{1}^{2} \sin ^{4} \theta\right)=C_{2}
\end{aligned}
$$

Since $C_{2}$ is constant, it has the same value for every $\theta$. We can evaluate it in center of the cell where $(z=0) \theta(0)=\theta_{m}$ and $\dot{\theta}=0$; thus, we obtain

$$
\begin{aligned}
& C_{2}=-\frac{1}{2} \cos ^{2} \theta_{m}\left(K_{2} \cos ^{2} \theta_{m}+K_{3} \sin ^{2} \theta_{m}\right)\left(\frac{\pi}{4 d}\right)^{2}+\frac{1}{2}\left(\frac{\chi_{a}}{\mu_{0}} B^{2}-\frac{2 f W}{a}\right) \sin ^{2} \theta_{m} \\
& +I n_{e}\left(1-\frac{N_{1}}{2} \sin ^{2} \theta_{m}+\frac{3}{8} N_{1}^{2} \sin ^{4} \theta_{m}\right)
\end{aligned}
$$

From Equation (26), we obtain

$$
\frac{d \theta}{d z}=\sqrt{\frac{Z\left(\theta, \theta_{m}\right)}{g(\theta)}}
$$


where

$$
\begin{aligned}
& Z\left(\theta, \theta_{m}\right)=-\frac{1}{2}\left[K_{2}\left(\cos ^{4} \theta_{m}-\cos ^{4} \theta\right)+K_{3}\left(\cos ^{2} \theta_{m} \sin ^{2} \theta_{m}-\cos ^{2} \theta \sin ^{2} \theta\right)\right]\left(\frac{\pi}{4 d}\right)^{2} \\
& +\left(\frac{\chi_{a}}{\mu_{0}} B^{2}-\frac{2 f W}{a}\right)\left(\sin ^{2} \theta_{m}-\sin ^{2} \theta\right) \\
& +\operatorname{In} \frac{N_{1}}{2}\left[-\left(\sin ^{2} \theta_{m}-\sin ^{2} \theta\right)+\frac{3}{4} N_{1}\left(\sin ^{4} \theta_{m}-\sin ^{4} \theta\right)\right]
\end{aligned}
$$

and

$$
g(\theta)=\frac{1}{2}\left(K_{1} \cos ^{2} \theta+K_{3} \sin ^{2} \theta\right)
$$

After integration, we get

$$
d=\int_{\theta_{0}}^{\theta_{m}} \sqrt{\frac{g(\theta)}{Z(\theta, \dot{\theta})}} d \theta
$$

We denote

$$
\sin \lambda=\frac{\sin \theta}{\sin \theta_{m}} \quad\left(\sin \lambda_{0}=\frac{\sin \theta_{0}}{\sin \theta_{m}}\right)
$$

and so we get

$$
d \theta=\frac{\sin \theta_{m} \sqrt{1-\sin ^{2} \lambda}}{\sqrt{1-\sin ^{2} \theta_{m} \sin ^{2} \lambda}} d \lambda
$$

Thus, Equation (31) becomes

$$
d=\int_{\lambda_{0}}^{\pi / 2} \sqrt{\frac{K_{1}+\left(K_{3}-K_{1}\right) \sin ^{2} \theta_{m} \sin ^{2} \lambda}{Y\left(\lambda, \theta_{m}\right)}} \times \frac{d \lambda}{\sqrt{1-\sin ^{2} \theta_{m} \sin ^{2} \lambda}}
$$

where

$$
\begin{aligned}
& Y\left(\lambda, \theta_{m}\right)=K_{2}\left[2-\sin ^{2} \theta_{m}\left(1+\sin ^{2} \lambda\right)\right]\left(\frac{\pi}{4 d}\right)^{2} \\
& -K_{3}\left[1-\sin ^{2} \theta_{m}\left(1+\sin ^{2} \lambda\right)\right]\left(\frac{\pi}{4 d}\right)^{2}+\left(\frac{\chi_{a}}{\mu_{0}} B^{2}-\frac{2 f W}{a}\right) \\
& +\operatorname{In}_{2} N_{1}\left[-1+\frac{3}{4} N_{1}\left(1+\sin ^{2} \lambda\right) \sin ^{2} \theta_{m}\right]
\end{aligned}
$$

For the Freedericksz transition, we have $\theta_{m} \rightarrow 0$ and we obtain

$$
\begin{gathered}
\frac{\pi}{2}-\lambda_{0}=\frac{d}{\sqrt{K_{1}}} \sqrt{\left(2 K_{2}-K_{3}\right)\left(\frac{\pi}{4 d}\right)^{2}+\left(\frac{\chi_{a}}{\mu_{0}} B_{c}^{2}-\frac{2 f W}{a}\right)-I N_{1} n_{e}} \\
\tan \lambda_{0}=\cot \left[\frac{d}{\sqrt{K_{1}}} \sqrt{\left(2 K_{2}-K_{3}\right)\left(\frac{\pi}{4 d}\right)^{2}+\left(\frac{\chi_{a}}{\mu_{0}} B_{c}^{2}-\frac{2 f W}{a}\right)-I N_{1} n_{e}}\right]
\end{gathered}
$$

where $B_{c}$ is the critical field for the Freedericksz transition.

We use the boundary conditions [35]

$$
\left.\left(K_{1} \cos ^{2} \theta_{o}+K_{3} \sin ^{2} \theta_{0}\right)\left(\frac{d \theta}{d z}\right)\right|_{z= \pm d}=A \sin \theta_{0} \cos \theta_{0}\left(1+2 \xi \sin ^{2} \theta_{0}\right)
$$

so

$$
\left.\left(\frac{d \theta}{d z}\right)\right|_{z= \pm d}=\frac{A \sin \theta_{0} \cos \theta_{0}\left(1+2 \xi \sin ^{2} \theta_{0}\right)}{K_{1}+\left(K_{3}-K_{1}\right) \sin ^{2} \theta_{0}}
$$

and from Equation (28), we obtain

$$
\left.\left(\frac{d \theta}{d z}\right)\right|_{z= \pm d}=\sqrt{\frac{Z\left(\theta_{0}, \theta_{m}\right)}{g\left(\theta_{0}\right)}}
$$


From Equations (39) and (40) we get

$$
\sqrt{\frac{Z\left(\theta_{0}, \theta_{m}\right)}{g\left(\theta_{0}\right)}}=\frac{A \sin \theta_{0} \cos \theta_{0}\left(1+2 \xi \sin ^{2} \theta_{0}\right)}{K_{1}+\left(K_{3}-K_{1}\right) \sin ^{2} \theta_{0}}
$$

Using $\lambda_{0}$ from Equation (32), Equation (41) becomes

$$
\frac{\sin \lambda_{0} \sqrt{1-\sin ^{2} \lambda_{0} \sin ^{2} \theta_{m}}}{\sqrt{K_{1}+\left(K_{3}-K_{1}\right) \sin ^{2} \lambda_{0} \sin ^{2} \theta_{m}}}=\cos \lambda_{0} \sqrt{Y\left(\lambda_{0}, \theta_{m}\right)}
$$

At the Freedericksz transition limit, the deviation angle is small—so $\theta_{m} \rightarrow 0$ —and we get

$$
\tan \lambda_{0}=\frac{\sqrt{K_{1}}}{A} \sqrt{\left(2 K_{2}-K_{3}\right)\left(\frac{\pi}{4 d}\right)^{2}+\left(\frac{\chi_{a}}{\mu_{0}} B_{c}^{2}-\frac{2 f W}{a}\right)-I N_{1} n_{e}}
$$

From Equations (37) and (43), we obtain an equation from which the critical field can be calculated:

$$
\cot \frac{E d}{\sqrt{K_{1}}}=\frac{\sqrt{K_{1}}}{A} E
$$

where

$$
E=\sqrt{\left(2 K_{2}-K_{3}\right)\left(\frac{\pi}{4 d}\right)^{2}+\left(\frac{\chi_{a}}{\mu_{0}} B_{c}^{2}-\frac{2 f W}{a}\right)-I N_{1} n_{e}}
$$

\section{Saturation Field}

When the applied field is much higher than the critical one, with weak anchoring on the solid walls, complete molecular reorientation is achieved. The minimum field for which this orientation appears is called the saturation field. In this case, $\theta \rightarrow \pi / 2$, so we get

$$
C_{1}=\cos ^{2} \theta\left(K_{2} \cos ^{2} \theta+K_{3} \sin ^{2} \theta\right) \dot{\varphi}=0
$$

and

$$
\left.I \frac{n_{0} n_{e}}{\sqrt{n_{0}^{2} \cos ^{2} \theta+n_{e}^{2} \sin ^{2} \theta}}=I n_{0}\left(1+\frac{N_{2}}{2} \cos ^{2} \theta+\frac{3 N_{2}^{2}}{8} \cos ^{4} \theta\right)\right)
$$

where

$$
N_{2}=1-\frac{n_{0}^{2}}{n_{e}^{2}}
$$

In this case, a prime integral for $\theta$ is

$$
\begin{aligned}
& \frac{1}{2}\left(K_{1} \cos ^{2} \theta+K_{3} \sin ^{2} \theta\right) \dot{\theta}^{2}+\frac{1}{2}\left(\frac{\chi_{a}}{\mu_{0}} B^{2}-\frac{f W}{a}\right) \sin ^{2} \theta+ \\
& +I n_{0}\left(1+\frac{N_{2}}{2} \cos ^{2} \theta+\frac{3 N_{2}^{2}}{8} \cos ^{4} \theta\right)=C_{2}
\end{aligned}
$$

As performed in the previous case, we can evaluate $C_{2}$ in the center of the cell where $z=0$; we have $\theta(0)=\theta_{m}$ and $\dot{\theta}=0$. Thus, we get

$$
\frac{1}{2}\left(\frac{\chi_{a}}{\mu_{0}} B^{2}-\frac{2 f W}{a}\right) \sin ^{2} \theta_{m}+\operatorname{In}\left(1+\frac{N_{2}}{2} \cos ^{2} \theta_{m}+\frac{3 N_{2}^{2}}{8} \cos ^{4} \theta_{m}\right)=C_{2}
$$


With this value, Equation (49) gives

$$
\frac{d \theta}{d z}=\sqrt{\frac{Z_{1}\left(\theta, \theta_{m}\right)}{g(\theta)}}
$$

where

$$
\begin{aligned}
& Z_{1}\left(\theta, \theta_{m}\right)=\frac{1}{2}\left(\frac{\chi_{a}}{\mu_{0}} B^{2}-\frac{2 f W}{a}\right)\left(\cos ^{2} \theta-\cos ^{2} \theta_{m}\right) \\
& +\frac{I n_{0} N_{2}}{2}\left[\left(\cos ^{2} \theta-\cos ^{2} \theta_{m}\right)+\frac{3 N_{2}}{4}\left(\cos ^{4} \theta-\cos ^{4} \theta_{m}\right)\right]
\end{aligned}
$$

so

$$
d=\int_{\theta_{0}}^{\theta_{m}} \sqrt{\frac{g(\theta)}{Z_{1}\left(\theta, \theta_{m}\right)}} d \theta
$$

We change the variable $\theta$ into $\eta$ by denoting

$$
\cos \eta=\frac{\cos \theta_{m}}{\cos \theta} \quad\left(\cos \eta_{0}=\frac{\cos \theta_{m}}{\cos \theta_{0}}\right)
$$

and we get

$$
d=\int_{0}^{\eta_{0}} \sqrt{\frac{K_{3}+\left(K_{1}-K_{2}\right) \frac{\cos ^{2} \theta_{m}}{\cos ^{2} \eta}}{\left(\frac{\chi a}{\mu_{0}} B^{2}-\frac{2 f W}{a}\right)-I N_{2} n_{0}\left[1+\frac{3}{4} N_{2} \cos ^{2} \theta_{m}\left(1+\frac{1}{\cos ^{2} \eta}\right)\right]}} \times \frac{d \lambda}{\sqrt{\cos ^{2} \eta-\cos ^{2} \theta_{m}}}
$$

For $\theta_{m} \rightarrow \pi / 2$, we get

$$
d=\frac{1}{2} \frac{\sqrt{K_{3}}}{\sqrt{\left(\frac{\chi_{a}}{\mu_{0}} B_{S}^{2}-\frac{2 f W}{a}\right)-I N_{2} n_{0}}} \ln \frac{1+\sin \eta_{0}}{1-\sin \eta_{0}}
$$

and so

$$
\sin \eta_{0}=\tanh \frac{d F}{2 \sqrt{K_{3}}}
$$

where

$$
F=\sqrt{\left(\frac{\chi_{a}}{\mu_{0}} B_{s}^{2}-\frac{2 f W}{a}\right)-I N_{2} n_{0}}
$$

Considering Equation (51),

$$
\left.\left(\frac{d \theta}{d z}\right)\right|_{z= \pm d}=\sqrt{\frac{Z_{1}\left(\theta_{0}, \theta_{m}\right)}{g\left(\theta_{0}\right)}}
$$

and using Equation (39), we get

$$
\frac{A \sin \theta_{0} \cos \theta_{0}\left(1+2 \xi \sin ^{2} \theta_{0}\right)}{K_{3}+\left(K_{1}-K_{2}\right) \cos ^{2} \theta_{0}}=\sqrt{\frac{Z_{1}\left(\theta_{0}, \theta_{m}\right)}{g\left(\theta_{0}\right)}}
$$

Using $\eta_{0}$ from (47), Equation (53) becomes

$$
\frac{A \sqrt{1-\frac{\cos ^{2} \theta_{m}}{\cos ^{2} \eta_{0}}\left[1+2 \xi\left(1-\frac{\cos ^{2} \theta_{m}}{\cos ^{2} \eta_{0}}\right)\right] \frac{1}{\cos \eta_{0}}}}{\sqrt{K_{3}+\left(K_{1}-K_{2}\right) \frac{\cos ^{2} \theta_{m}}{\cos ^{2} \eta_{0}}}}=\sqrt{G\left(\eta_{0}, \theta_{m}\right)}
$$


where

$$
G\left(\eta_{0}, \theta_{m}\right)=\left(\frac{1}{\cos ^{2} \eta_{0}}-1\right)\left\{\left(\frac{\chi_{a}}{\mu_{0}} B^{2}-\frac{2 f W}{a}\right)-I N_{2} n_{0}\left[1+\frac{3}{4}\left(1+\frac{1}{\cos ^{2} \eta}\right) \cos ^{2} \theta_{m}\right]\right\}
$$

Using $\theta_{m} \rightarrow \pi / 2$, we get

$$
\sin \eta_{0}=\frac{A(1+2 \xi)}{F \sqrt{K_{3}}}
$$

From Equations (57) and (63), we get

$$
\operatorname{coth} \frac{d F}{2 \sqrt{K_{3}}}=\frac{\sqrt{K_{3}} F}{A(1+2 \xi)}
$$

Thus, from Equation (64), we can evaluate the saturation field $B_{s}$ using Equations (58) for the $F$ function.

As magnetic nanoparticles, we used $W=5 \times 10^{-9} \mathrm{~N} / \mathrm{m}, a=10^{-8} \mathrm{~m}$. The volumetric fraction was taken as $f=0.1 \%$ and the cell thickness was $2 d=200 \mu \mathrm{m}$. We chose an electromagnetic energy density in the range $10^{-3}-10^{-4} \mathrm{~J} / \mathrm{m}^{3}$. For the studied configuration, the electric field component of the laser beam was parallel to the glass plate (Figure 1).

The liquid crystals considered as hosts for the magnetic nanoparticles were chosen for their different magnetic anisotropy values. As can be seen from Table 1, 5CB had positive values for magnetic anisotropy, while $\mathrm{CCN}-37$ had negative anisotropy.

Table 1. Material parameters for nematic compounds $5 \mathrm{CB}$ and $\mathrm{CCN}-37$ estimated from the results given in $[32,37]$.

\begin{tabular}{ccc}
\hline Constants & $5 \mathbf{C B}$ & $\mathbf{C C N}-37$ \\
\hline$\chi_{a}$ & $1.46 \times 10^{-6}$ & $-0.95 \times 10^{-7}$ \\
$K_{1}$ & $6.2 \mathrm{pN}$ & $4.6 \mathrm{pN}$ \\
$K_{2}$ & $3.9 \mathrm{pN}$ & $4.1 \mathrm{pN}$ \\
$K_{3}$ & $8.2 \mathrm{pN}$ & $9.3 \mathrm{pN}$ \\
$n_{0}$ & 1.544 & 1.5 \\
$n_{e}$ & 1.736 & 1.53 \\
\hline
\end{tabular}

\section{Results and Discussions}

For the 5CB host ferronematic, which has both positive magnetic and dielectric anisotropies, the obtained plots are given in Figures 2 and 3.

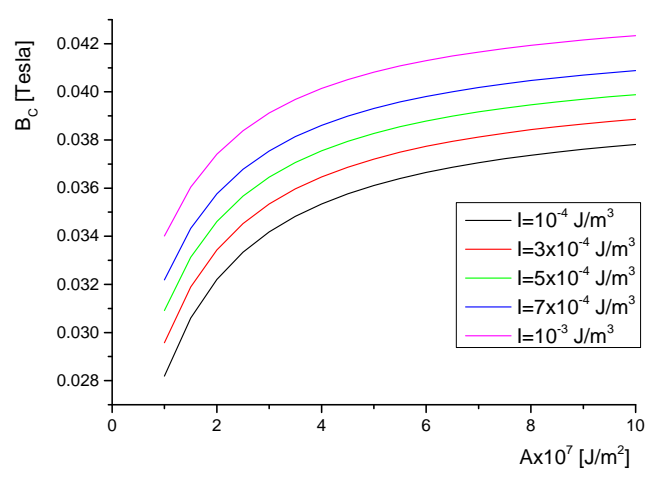

(a)

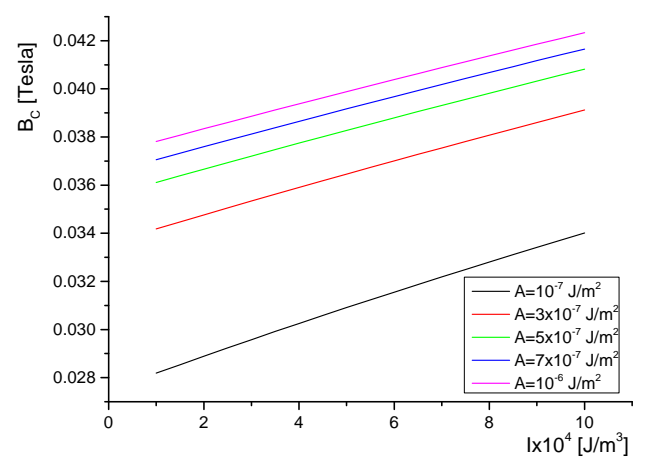

(b)

Figure 2. Critical field for ferromagnetic nanoparticle suspensions in 5CB: (a) the critical field versus anchoring strength plot and (b) the critical field versus laser intensity. 


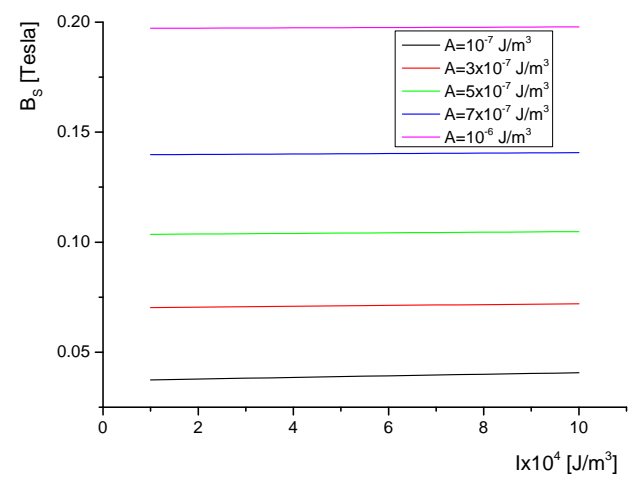

Figure 3. Saturation field for ferromagnetic nanoparticle suspensions in 5CB.

As can be seen from Figure 2a,b, there is a large increase of the critical field with the increase of the laser beam intensity. The Freedericksz transition can be experimentally observed by a laser beam crossing through the sample. If the laser beam intensity is sufficiently high, its electric component may induce an electrical Freedericksz transition, opposed to the one induced by the applied magnetic field. Thus, when the laser intensity is increased, a higher magnetic field is required to obtain the molecular orientation, so the critical field increases.

In Figure 3 , we observe a slow increase of the saturation field with the laser beam intensity. This effect can be explained by the high magnetic field values at which this effect appears. Thus, the laser intensity cannot induce an electric Freedericksz transition which is sufficiently strong to compensate the magnetic transition produced by the applied field, especially when some of the molecules are attached on ferromagnetic nanoparticles which are more easily aligned by the field. A much larger effort is required for the magnetic field to compensate the anchoring strength, so an increase of the saturation field with the anchoring energy is observed. The distance between $B_{S}$ lines is larger for higher values of anchoring strength.

For the CCN-37 nematic, which has a negative magnetic anisotropy, the magnetic field applied must be parallel to the nematic director. For a twisted alignment, the field must be parallel to the solid walls and the laser perpendicular to the walls, as shown in Figure 4. Thus, some changes are required when evaluating the free energy density component related to the interaction between the nematic molecules and magnetic field. Thus,

$$
f_{v 2}=\frac{\chi_{a}}{2 \mu_{0}} B^{2} \cos ^{2} \theta=\frac{\chi_{a}}{2 \mu_{0}} B^{2}\left(1-\sin ^{2} \theta\right)=\frac{\chi_{a}}{2 \mu_{0}} B^{2}-\frac{\chi_{a}}{2 \mu_{0}} B^{2} \sin ^{2} \theta=\frac{\chi_{a}}{2 \mu_{0}} B^{2}+\frac{\left|\chi_{a}\right|}{2 \mu_{0}} B^{2} \sin ^{2} \theta
$$

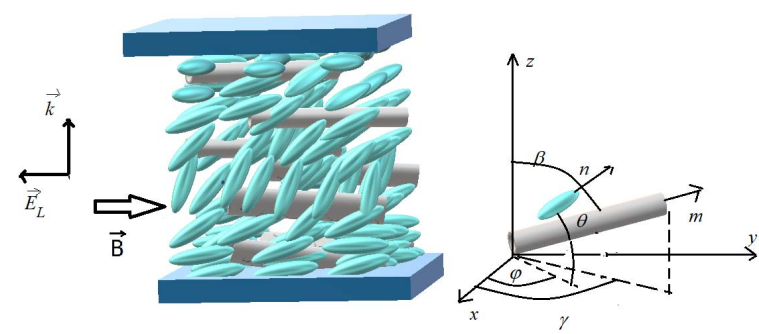

Figure 4. Molecular orientation of ferronematic and laser beam when CCN-37 is used as host.

From this equation, we shall only keep the second term because the first one is constant and has no influence on Euler-Lagrange equations. 
For the interaction of the nematic molecules with the inserted ferroparticles, the free energy density becomes

$$
f_{v 4}=\frac{f W}{a}(\vec{m} \vec{n})^{2}=\frac{f W}{a} \cos ^{2}(\theta-\beta)=\frac{f W}{a}\left[1-\sin ^{2}(\theta-\beta)\right]
$$

Using the same procedure and writing the Euler-Lagrange equation for $\beta$, we finally obtain

$$
\frac{f W}{a}(\vec{m} \vec{n})^{2}=\frac{f W}{a}-\frac{f W}{a} \sin ^{2} \theta
$$

The critical fields and saturation field can be written as a function of $E$ given by Equation (45) and as a function of $F$ given by Equation (51); where we must replace $\chi_{a}$ with $\left|\chi_{a}\right|$ and $f w / a$ with $-f w / a$.

The plots obtained with these parameters are given in Figure 5. As can be observed from Figure $6 a, b$, the critical field decreases with the laser beam intensity because of this "competition" between the magnetic effect on the nanoparticle and the electric and magnetic effect on liquid crystal molecules. As with all the previous cases, the saturation field is strongly affected by the anchoring energy (Figure 5) because complete reorientation is harder to achieve for rigid anchoring.

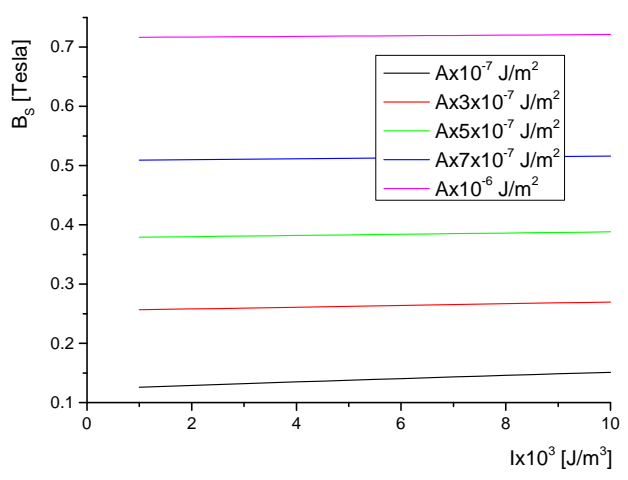

Figure 5. Saturation field for the CCN-37 based ferronematic.

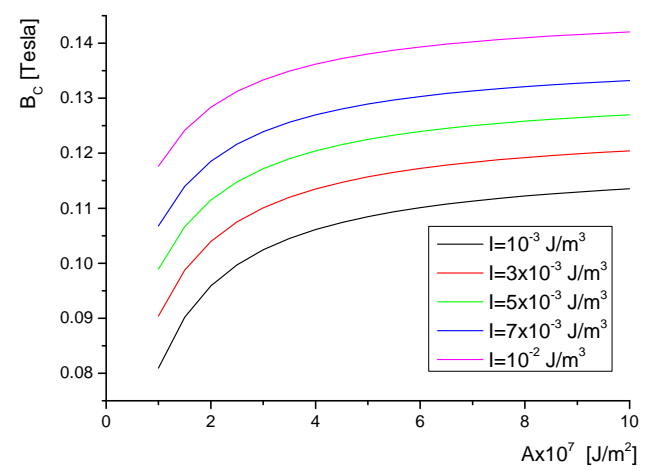

(a)

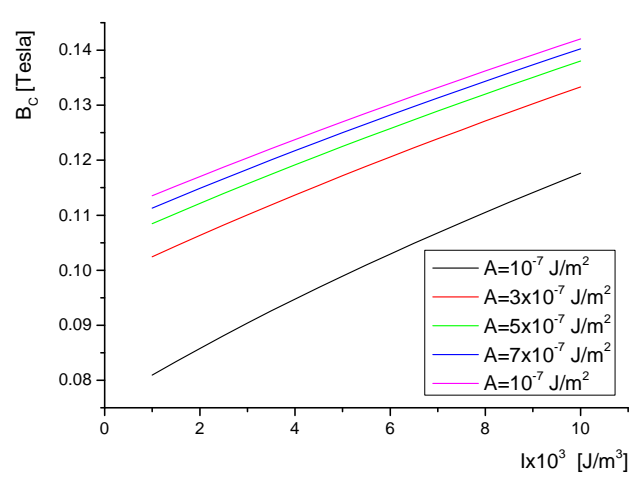

(b)

Figure 6. Critical field for the CCN-37 based ferronematic: (a) the critical field versus anchoring strength plot and (b) the critical field versus laser intensity.

\section{Conclusions}

The influences of the anchoring forces and laser beam are significant on the critical field and saturation field in ferronematics and should not be neglected. By choosing the proper combination of the liquid crystal anisotropy, inserted nanoparticles and surface anchoring, the critical field can be 
considerably decreased. The saturation field can also be adjusted by the light intensity used for LC-cell commands. Thus, the molecular reorientation can be efficiently controlled, and the efficacy of LCs for applications such as in LCDs or phase modulators can be improved.

Author Contributions: Conceptualization, C.C. and E.P.; methodology, E.P. and G.I.; software, E.P. and C.C.; validation, C.C., G.I. and E.P.; formal analysis, G.I.; investigation, C.C. and E.P.; resources, C.C. and G.I.; data curation, C.C. and E.P.; writing-original draft preparation, C.C.; writing-review and editing, C.C., G.I. and E.P.; visualization, G.I.; supervision, E.P.; project administration, C.C.; funding acquisition, C.C. All authors have read and agreed to the published version of the manuscript.

Funding: This research was funded by the Romanian Governmental Representative at JINR Dubna through the scientific project "The study of carbon nanotubes self-assembling in a nematic host", JINR order nr.397/27.05.2019 item 8 . The cost of the article's publication was funded by the University Politehnica of Bucharest through the PubArt project.

Acknowledgments: In this section you can acknowledge any support given which is not covered by the author contribution or funding sections. This may include administrative and technical support, or donations in kind (e.g., materials used for experiments).

Conflicts of Interest: The authors declare no conflict of interest.

\section{References}

1. Koch, K.; Kundt, M.; Eremin, A.; Nadasi, H.; Schmidt, A.M. Efficient ferronematic coupling with polymer-brush particles. Phys. Chem. Chem. Phys. 2020, 22, 2087-2097. [CrossRef] [PubMed]

2. Romero-Hasler, P.; Martinez-Miranda, L.J.; Meneses-Franco, A.; Soto-Bustamante, E.A. $\mathrm{TiO}_{2}$ nanoparticleliquid crystal interaction with smectogenic monomers and their electropolymerised polymers. Liq. Cryst. 2019. [CrossRef]

3. Saravanan, M. Director deformation in a nematic liquid crystal with a ferromagnetic nanoparticle suspension. Chin. J. Phys. 2019, 59, 426-433. [CrossRef]

4. Iabobescu, G.E. Freedericksz transitions of twisted ferronematics in magnetic field. J. Magn. Magn. Mater. 2018, 468, 65-68. [CrossRef]

5. Saravanan, M.; Ameya, D.J.; Murthy, A.S.V. Perturbed soliton and director deformation in a ferronematic liquid crystal. Chaos Soliton Fract. 2018, 106, 220-226.

6. Petrescu, E.; Cirtoaje, C. Dynamic behavior of a nematic liquid crystal mixed with $\mathrm{CoFe}_{2} \mathrm{O}_{4}$ ferromagnetic nanoparticles in a magnetic field. Beilstein J. Nanotech. 2017, 8, 2467-2473. [CrossRef]

7. Bury, P.; Vevericik, M.; Kopcansky, P.; Timko, M.; Zavisova, V. Effect of spherical, rod-like and chain-like magnetic nanoparticles on magneto-optical response of nematics. Acta Phys. Pol. A 2019, 136, 101-106. [CrossRef]

8. Petrescu, E.; Bena, E.R.; Citoaje, C. Polarization gratings using ferronematics-An elastic continuum theory. J. Magn. Magn. Mater. 2013, 336, 44-348. [CrossRef]

9. Burylov, S.V.; Raikher, Y.L. Magnetic Fredericksz transition in a ferronematic. J. Magn. Magn. Mater. 1993, 122, 62-65. [CrossRef]

10. Brochard, F.; De Gennes P. G. Theory of magnetic suspensions in liquid crystals. J. Phys. France 1970, 31, 691-708. [CrossRef]

11. Makarov, D.V.; Novikov, A.A.; Zakhlevnykh, A.N. Ferrocholesteric-ferronematic transitions induced by shear flow and magnetic field. Beilstein J. Nanotech. 2017, 8, 2552-2561. [CrossRef] [PubMed]

12. Zakhlevnykh, A.N.; Petrov, D.A. Weak coupling effects and re-entrant transitions in ferronematic liquid crystals. J. Mol. Liq. 2014, 198, 223-233. [CrossRef]

13. Burylov, S.V.; Raikher, Y.L. Orientation of a solid particle embedded in a monodomain nematic liquid-crystal. Phys. Rev. E 1994, 50, 358-367. [CrossRef] [PubMed]

14. Cirtoaje, C.; Motoc, C.; Petrescu, E.; Bena R.E. New magnetic methods for determination of elastic constants and rotational viscosity coefficient in nematic liquid crystals. Univer. Politeh. Buch. Ser. A 2010, 72, 233-246.

15. Petrov, D.A.; Skokov, P.K.; Zakhlevnykh, A.N. Magnetic field induced orientational transitions in liquid crystals doped with carbon nanotubes. Beilstein J. Nanotech. 2017, 8, 2807-2817. [CrossRef]

16. Jimenez-Marin, E.; Villalpando, I.; Trejo-Valdez, M.; Cervantes-Sodi, F.; Vargas-Garcia, J.R.; Torres-Torres, C. Coexistence of positive and negative photoconductivity in nickel oxide decorated multiwall carbon nanotubes. Mat. Sci. Eng. B Solid 2017, 1220, 22-29. [CrossRef] 
17. Chang, C.; Zhao, Y.; Liu, Y.; An, L. Liquid crystallinity of carbon nanotubes. RSC Adv. 2018, 8, 15780-15795. [CrossRef]

18. Petrescu, E.; Cirtoaje, C. Dynamic behavior of a nematic liquid crystal with added carbon nanotubes in an electric field. Beilstein J. Nanotech. 2018, 9, 233-241. [CrossRef]

19. Jain, A.K.; Deshmukh, R.R. Electro-optical and dielectric study of multi-walled carbon nanotube doped polymer dispersed liquid crystal films. Liq. Cryst. 2019, 8, 1191-1202. [CrossRef]

20. Ara, M.H.M.; Dehghani, Z. Improvement of the third order nonlinear optical properties of nematic liquid crystal under the influence of different compositional percentage of doped SWCNT and the external electric field. J. Mol. Liq. 2019, 275, 281-289.

21. Cetinkaya, M.C.; Yildiz, S.; Ozbek, H. The effect of -COOH functionalized carbon nanotube doping on electro-optical, thermo-optical and elastic properties of a highly polar smectic liquid crystal. J. Mol. Liq. 2018, 272, 801-2814. [CrossRef]

22. Lisetski, L.N.; Fedoryako, A.P.; Samoilov, A.N.; Minenko, S.S.; Soskin, M.S.; Lebovka, N.I. Optical transmission of nematic liquid crystal $5 \mathrm{CB}$ doped by single-walled and multi-walled carbon nanotubes. Eur. J. Phys. 2014, 37, 68. [CrossRef] [PubMed]

23. Atasiei, R.; Dascalu, C.; Raicopol, M. The time dependence of the electric charge in a nematic cell aligned with doped polypyrrole. Univer. Politeh. Buc. Ser. A 2013, 75, 293-298.

24. Volpati, D.; Massey, M.K.; Johnson, D.W.; Kotsialos, A.; Qaiser, F.; Pearson, C.; Coleman, K.S.; Tiburzi, G.; Zeze, D.A.; Petty, M.C. Exploring the alignment of carbon nanotubes dispersed in a liquid crystal matrix using coplanar electrodes. J. Appl. Phys. 2015, 117, 125303. [CrossRef]

25. Denktas, C.; Ocak, H.; Okutan, M.; Yildiz, A.; Eran, B.B.; Koysal, O. Effect of multi wall carbon nanotube on electrical properties 4-[4-((S)-Citronellyloxy)benzoyloxy]benzoic acid liquid crystal host. Compos. Part B Eng. 2015, 82, 173-177. [CrossRef]

26. Bale, S.; Liyana-Arachchi, T.P.; Hung, F.R. Molecular dynamics simulation of single-walled carbon nanotubes inside liquid crystals. Mol. Simulat. 2016, 42, 1242-1248. [CrossRef]

27. Yadav, S.P.; Singh, S. Carbon nanotube dispersion in nematic liquid crystals: An overview. Prog. Mater. Sci. 2016, 80, 38-176. [CrossRef]

28. Kedzierski, K.; Rytel, K.; Barszcz, B.; Gronostaj, A.; Majchrzycki, L.; Wrobel, D. On the temperature dependent electrical resistivity of CNT layers in view of Variable Range Hopping models. Org. Electron. 2017, 43, 253-261. [CrossRef]

29. Palarie, I.; Dascalu, C.; Iacobescu, G.E. Controlling the orientation of microgrooves and the depth of the ripple structure in dye-doped liquid crystal cells. Liq. Cryst. 2010, 37, 195-199. [CrossRef]

30. Staic, M.; Petrescu-Nita, A. Symmetry group of two special types of carbon nanotori. . Acta Crystallogr. 2013, 69, 435-439. [CrossRef]

31. Assanto, G.; Alberucci, A.; Piccardi, A. Nematicons. In Nematicons: Spatial Optical Solitons in Nematic Liquid Crystals; Assanto, G., Ed.; Wiley: Hoboken, NJ, USA, 2012.

32. Ian Stewart, W. The Static and Dinamic Continuum Theory of Liquid Crystals a Mathematical Introduction; Taylor \& Francis: London, UK; New York, NY, USA, 2004; p. 330.

33. Rapini, A.; Papoular, M. Distorsion d'une lamelle nématique sous champ magnétique conditions d'ancrage aux parois. J. Phys. Colloques 1969, 30, C4-54-C4-56. [CrossRef]

34. Guochen, Y.; Jianru, S.; Ying, L. Surface anchoring energy and the first order Freedericksz transition of a NLC cell. Liq. Cryst. 2000, 27, 875-882. [CrossRef]

35. Oswald, P.; Pieranski, P. Nematic and Cholesteric Liquid Crystals; Taylor \& Francis Group: Oxfordshire, UK, 2005; pp. 157-158.

36. Cirtoaje, C.; Petrescu, E.; Stan, C.; Creanga, D. Ferromagnetic nanoparticles suspensions in twisted nematic, Physica E 2016, 79, 38-43. [CrossRef]

37. Oswald, P.; Poy, G.; Dequidt, A. Lehmann rotation of twisted bipolar cholesteric droplets: Role of Leslie, Akopyan and Zel'dovich thermomechanical coupling terms of nematodynamics. Liq. Cryst. 2016, 44, 969-988. [CrossRef]

(C) 2020 by the authors. Licensee MDPI, Basel, Switzerland. This article is an open access article distributed under the terms and conditions of the Creative Commons Attribution (CC BY) license (http://creativecommons.org/licenses/by/4.0/). 